BULLETIN (New Series) OF THE

AMERICAN MATHEMATICAL SOCIETY

Volume 48, Number 4, October 2011, Pages 497-501

S 0273-0979(2011)01343-X

Article electronically published on June 10, 2011

\title{
POINCARÉ RECURRENCE AND NUMBER THEORY: THIRTY YEARS LATER
}

\author{
BRYNA KRA
}

Immediately following the commentary below, this previously published article is reprinted in its entirety: H. Furstenberg, Poincaré recurrence and number theory, Bull. Amer. Math. Soc. (N.S.) 5 (1981), no. 3, 211-234.

Hillel Furstenberg's 1981 article in the Bulletin of the American Mathematical Society gives an elegant introduction to the interplay between dynamics and number theory, summarizing the major developments that occurred in the few years after his landmark paper 21. The field has evolved over the past thirty years, with major advances on the structural analysis of dynamical systems and new results in combinatorics and number theory. Furstenberg's article continues to be a beautiful introduction to the subject, drawing together ideas from seemingly distant fields.

Furstenberg's article [21] gave a general correspondence between regularity properties of subsets of the integers and recurrence properties in dynamical systems, now dubbed the Furstenberg Correspondence Principle. He then showed that such recurrence properties always hold, proving what is now referred to as the Multiple Recurrence Theorem. Combined, these results gave a new proof of Szemerédi's Theorem [45]: if $S \subset \mathbb{Z}$ has positive upper density, then $S$ contains arbitrarily long arithmetic progressions. This proof led to an explosion of activity in ergodic theory and topological dynamics, beginning with new proofs of classic results of Ramsey Theory and ultimately leading to significant new combinatorial and number theoretic results. The full implications of these connections have yet to be understood.

The approach harks back to the earliest results on recurrence, in the measurable setting and in the topological setting. A measure preserving system is a quadruple $(X, \mathcal{B}, \mu, T)$, where $X$ denotes a set, $\mathcal{B}$ is a $\sigma$-algebra on $X, \mu$ is a probability measure on $(X, \mathcal{B})$, and $T: X \rightarrow X$ is a measurable transformation such that $\mu\left(T^{-1}(A)\right)=\mu(A)$ for all $A \in \mathcal{B}$. Poincaré Recurrence states that if $(X, \mathcal{B}, \mu, T)$ is a measure preserving system and $A \in \mathcal{B}$ with $\mu(A)>0$, there exists $n \in \mathbb{N}$ such that $\mu\left(A \cap T^{-n} A\right)>0$. A (topological) dynamical system is a pair $(X, T)$, where $X$ is a compact metric space and $T: X \rightarrow X$ is a continuous map. One can show that any such topological space admits a Borel probability measure that preserves $T$. In particular, Poincaré Recurrence implies recurrence in the topological setting. Birkhoff 13 gave a direct proof of this, showing that in any dynamical system

Received by the editors May 16, 2011.

2010 Mathematics Subject Classification. Primary 37A05.

The author was partially supported by NSF grant 0900873.

(C)2011 American Mathematical Society Reverts to public domain 28 years from publication 
$(X, T)$, there exists $x \in X$ such that $T^{n_{k}} x \rightarrow x$ for some sequence of integers $n_{k} \rightarrow \infty$.

Birkhoff's recurrence was generalized by Furstenberg and Weiss [26], who showed that given a dynamical system $(X, T)$ and an integer $\ell \geq 1$, there exists $x \in X$ such that $T^{n_{k}} x \rightarrow x, T^{2 n_{k}} x \rightarrow x, \ldots, T^{\ell n_{k}} x \rightarrow x$ for some sequence of integers $n_{k} \rightarrow \infty$. Using an analog of the Furstenberg Correspondence Principle adapted to topological dynamical systems, this in turn implies van der Waerden's Theorem: in any finite partition of the integers, some piece contains arbitrarily long arithmetic progressions. Topological methods were then used to prove numerous other partition results, including Schur's Theorem, a multidimensional version of van der Waerden's Theorem, and the Hales-Jewett Theorem. As a sample of these techniques, Furstenberg gives a proof of a polynomial result, shown independently by Sàrkőzy [44]: in any finite partition of the integers, some piece contains two integers which differ by a square. Vast generalizations of such polynomial results were given by Bergelson and Leibman ([7] and 8]).

In the measure theoretic setting, Furstenberg proved a far-reaching generalization of the Poincaré Recurrence Theorem in his Multiple Recurrence Theorem: if $(X, \mathcal{B}, \mu, T)$ is a measure preserving system, $\ell \geq 1$ is an integer, and $A \in \mathcal{B}$ has positive measure, then there exists $n \in \mathbb{N}$ such that

$$
\mu\left(A \cap T^{-n} A \cap T^{-2 n} A \cap \cdots \cap T^{-\ell n} A\right)>0 .
$$

The study of which iterates $n$ are possible has received significant attention (polynomial iterates [7, generalized polynomials 12, sequences arising from HardyFields [17, shifts of the primes [7] and [18]), and this result has been generalized in numerous other ways (see for example [25] and 40]). Many of these results have yet to be proved using methods that do not rely on dynamics.

Via the Furstenberg Correspondence Principle, the analog of equation (11) with the transformations $T, T^{2}, \ldots, T^{\ell}$ replaced by commuting transformations $T_{1}, T_{2}$, $\ldots, T_{\ell}$ leads to a multidimensional Szemerédi Theorem, and this and generalizations were proven by Furstenberg and Katznelson ([23] and [24]). This too has been studied further, including restrictions on iterates (see [7, [11, and [19]) and generalizations to other groups (for example [41]). Again, many of these results have yet to be proven via combinatorial methods.

Using ergodic theory, the natural approach to prove positivity of an expression such as that in equation (11) is to take the average for $1 \leq n \leq N$ and show that the $\lim$ inf of this average is positive as $N \rightarrow \infty$. More generally, one can consider convergence of averages

$$
\frac{1}{N} \sum_{n=1}^{N} f_{1}\left(T_{1}^{a_{1}(n)} x\right) f_{2}\left(T_{2}^{a_{2}(n)} x\right) \cdots f_{\ell}\left(T_{\ell}^{a_{\ell}(n)} x\right),
$$

where $(X, \mathcal{B}, \mu)$ is a probability space; $T_{1}, T_{2}, \ldots, T_{\ell}: X \rightarrow X$ are commuting, measure preserving transformations; $f_{1}, f_{2}, \ldots, f_{\ell} \in L^{\infty}(\mu)$; and the exponents $a_{1}(n), a_{2}(n), \ldots, a_{\ell}(n)$ are sequences of integers. The existence of the limit in $L^{2}(\mu)$ and the study of the structures controlling the limiting behavior of such averages have received much attention within ergodic theory and have more recently led to new number theoretic results. The case when all the transformations $T_{i}$ are equal with linear exponents is fully understood, with partial results (for example in [21 and [15]) and the complete convergence (in [32]). These results 
have been generalized and viewed in other ways, with further studies of the linear case [49], polynomial iterates ([27], [33] and [41]), commuting transformations ([16] and [46]), restrictions on the iterates for commuting transformations (see for example [36], [14, 1], and [2]), nilpotent group actions [9], and the corresponding average for flows (see [4], 10] and [3]). For a single transformation, we have a complete understanding in 32 of the structures controlling convergence (with a topological analog in 35]). The general convergence and associated structures controlling such averages are yet to be understood.

The connections to number theoretic and combinatorial problems continue to grow, particularly with the spectacular breakthrough of Green and Tao 28] showing that the primes contain arbitrarily long arithmetic progressions. While there is no explicit use of ergodic theory in Green and Tao's proof, the methods used by Furstenberg 21] influence the approach. In more recent work on asymptotics of the number of progressions in the primes ([29, 30], and 31]) and other connections between ergodic theory and number theory (see, for example [6], [34, and [20]), the structures controlling the averages of the form in equation (2) play a prominent role.

This is only a brief overview of current areas of research with origins in the work surveyed in Furstenberg's 1981 Bulletin article. An extensive introduction to the field is contained in Furstenberg's book 22. There are more recent surveys on recurrence, ergodic Ramsey Theory, convergence problems, and connections to number theory (see for example [4, [5, 42, 37, 38, 39, [47, and 48]) that are natural continuations of the topics reviewed in Furstenberg's 1981 article.

\section{REFERENCES}

[1] T. Austin. Pleasant extensions retaining algebraic structure, I. arXiv:0905.0518

[2] T. Austin. Pleasant extensions retaining algebraic structure, II. arXiv:0910.0907

[3] T. Austin. Norm convergence of continuous-time polynomial multiple ergodic averages. arXiv:1103.0223v2

[4] V. Bergelson. Combinatorial and Diophantine applications of ergodic theory (with appendices by A. Leibman and by A. Quas and M. Wierdl). Handbook of Dynamical Systems, Vol. 1B, (B. Hasselblatt, A. Katok, Eds.), Elsevier, Amsterdam, 2006, 745-841. MR 2186252 (2006j:37006)

[5] V. Bergelson. Ergodic Ramsey theory - an update. Ergodic Theory of $\mathbb{Z}^{d}$-actions, (M. Pollicott, K. Schmidt, Eds.), London Math. Soc. Lecture Note Ser., 228, Cambridge University Press, Cambridge, 1996, 1-61. MR.1411215 (98g:28017)

[6] V. Bergelson, B. Host, B. Kra, with an appendix by I. Ruzsa. Multiple recurrence and nilsequences. Invent. Math., 160 (2005), 261-303. MR2138068(2007i:37009)

[7] V. Bergelson, A. Leibman. Polynomial extensions of van der Waerden's and Szemerédi's theorems. Jour. Amer. Math. Soc. 9 (1996), 725-753. MR1325795 (96j:11013)

[8] V. Bergelson, A. Leibman. Set-polynomials and polynomial extension of the Hales-Jewett theorem. Ann. of Math. 150 (1999), 33-75. MR.1715320 (2000h:05226)

[9] V. Bergelson, A. Leibman. A nilpotent Roth theorem. Invent. Math. 147 (2002), 429-470. MR.1881925 (2003a:37002)

[10] V. Bergelson, A. Leibman, C. G. Moreira. From discrete to continuous time ergodic theorems. Preprint.

[11] V. Bergelson, A. Leibman, T. Ziegler. The shifted primes and the multidimensional Szemerédi and polynomial van der Waerden theorems. To appear, Comptes Rendus Math.

[12] V. Bergelson, R. McCutcheon. Idempotent ultrafilters, multiple weak mixing and Szemerédi's theorem for generalized polynomials. J. Analyse Math. 111 (2010), 77-130. MR2747062

[13] G. D. Birkhoff. Dynamical Systems. Amer. Math. Soc. Colloq. Publ. 9 American Mathematical Society, Providence, 1927. 
[14] Q. Chu, N. Frantzikinakis, B. Host. Ergodic averages of commuting transformations with distinct degree polynomial iterates. To appear, Proc. Lond. Math. Soc.

[15] J.-P. Conze, E. Lesigne. Théorèmes ergodiques pour des mesures diagonales. Bull. Soc. Math. France 112 (1984), 143-175. MR788966 (86i:28019)

[16] J.-P. Conze, E. Lesigne. Sur un théorème ergodique pour des mesures diagonales. Publ. Inst. Rech. Math. Rennes 1987-1 (1988), 1-31. MR939438(89e:22012)

[17] N. Frantzikinakis. Multiple recurrence and convergence for Hardy sequences of polynomial growth. J. Analyse Math. 112 (2010), 79-135. MR2762998

[18] N. Frantzikinakis, B. Host, B. Kra. Multiple recurrence and convergence for sets related to the primes. J. Reine Angew. Math., 611 (2007), 131-144. MR2361086 (2009c:11023)

[19] N. Frantzikinakis, B. Host, B. Kra. The polynomial multidimensional Szemerédi Theorem along shifted primes. arXiv:1009.1484

[20] N. Frantzikinakis, E. Lesigne, M. Wierdl. Powers of sequences and recurrence. Proc. London. Math. Soc., 98 (2009), 504-530. MR2481957(2009m:37013)

[21] H. Furstenberg. Ergodic behavior of diagonal measures and a theorem of Szemerédi on arithmetic progressions. J. Analyse Math. 31 (1977), 204-256. MR0498471 (58:16583)

[22] H. Furstenberg. Recurrence in Ergodic Theory and Combinatorial Number Theory. Princeton University Press, Princeton, 1981. MR603625 (82j:28010)

[23] H. Furstenberg, Y. Katznelson. An ergodic Szemerédi theorem for commuting transformations. J. Analyse Math. 34 (1978), 275-291. MR531279 (82c:28032)

[24] H. Furstenberg, Y. Katznelson. An ergodic Szemerédi theorem for IP-systems and combinatorial theory. J. Analyse Math. 45 (1985), 117-168 MR833409 (87m:28007)

[25] H. Furstenberg, Y. Katznelson. A density version of the Hales-Jewett theorem. J. Analyse Math. 57 (1991), 64-119. MR 1191743 (94f:28020)

[26] H. Furstenberg, B. Weiss. Topological dynamics and combinatorial number theory. J. Analyse Math. 34 (1978), 61-85. MR.531271 (80g:05009)

[27] H. Furstenberg, B. Weiss. A mean ergodic theorem for $\frac{1}{N} \sum_{n=1}^{N} f\left(T^{n} x\right) \mathrm{g}\left(T^{n^{2}} x\right)$. Convergence in Ergodic Theory and Probability (Columbus, OH, 1993), Walter de Gruyter, 1996, 193-227. MR.1412607 (98e:28019)

[28] B. Green, T. Tao. The primes contain arbitrarily long arithmetic progressions. Ann. of Math., 167 (2008), 481-547. MR2415379 (2009e:11181)

[29] B. Green, T. Tao. Linear equations in the primes. Ann. of Math., 171 (2010), 1753-1850. MR2680398

[30] B. Green, T. Tao. The Möbius function is strongly orthogonal to nilsequences. To appear, Ann. of Math.

[31] B. Green, T. Tao, T. Ziegler. An inverse theorem for the Gowers $U^{k}$ norm. arXiv:1009.3998.

[32] B. Host, B. Kra. Nonconventional averages and nilmanifolds. Ann. of Math., 161 (2005), 398-488. MR2150389 (2007b:37004)

[33] B. Host, B. Kra. Convergence of Polynomial ergodic averages. Isr. J. Math., 149 (2005), 1-19. MR2191208 (2007c:37004)

[34] B. Host, B. Kra. Uniformity norms on $\ell^{\infty}$ and applications. J. Analyse Math., 108 (2009), 219-276. MR2544760 (2010j:11018)

[35] B. Host, B. Kra, A. Maass. Nilsequences and a structure theorem for topological dynamical systems. Adv. in Math., 224 (2010), 103-129. MR.2600993

[36] M. Johnson. Convergence of polynomial ergodic averages. Illinois J. Math., 53 (2009), 865882. MR2727359

[37] B. Kra. The Green-Tao Theorem on arithmetic progressions in the primes: an ergodic point of view. Bull. Amer. Math. Soc., 43 (2006), 3-23. MR.2188173 (2006h:11113)

[38] B. Kra. From combinatorics to ergodic theory and back again. Proceedings of the International Congress of Mathematicians, Madrid 2006, Vol. III, 57-76. MR2275670 (2007m:37014)

[39] B. Kra. Ergodic methods in additive combinatorics. Additive combinatorics, CRM Proc. Lecture Notes 43, Amer. Math. Soc., Providence, RI (2007), 103-144. MR2359470 (2008k:37018)

[40] A. Leibman. Multiple recurrence theorem for measure preserving actions of a nilpotent group. Geom. Funct. Anal., 8 (1998), 853-931. MR1650102 (99k:28019)

[41] A. Leibman. Convergence of multiple ergodic averages along polynomials of several variables. Isr. J. Math., 146 (2005), 303-316. MR2151605 (2006c:28016)

[42] R. McCutcheon. Elemental methods in ergodic Ramsey theory. Lecture Notes in Mathematics 1722. Springer-Verlag, Berlin, 1999. MR1738544 (2001c:05141) 
POINCARÉ RECURRENCE AND NUMBER THEORY: THIRTY YEARS LATER

[43] A. Potts. Multiple ergodic averages for flows and an application. To appear, Illinois J. Math.

[44] A. Sàrkőzy. On difference sets of integers III. Acta Math. Acad. Sci. Hungar., 31 (1978), 125-149. MR0466059 (57:5942)

[45] E. Szemerédi. On sets of integers containing no $k$ elements in arithmetic progression. Acta Arith. 27 (1975) 199-245. MR0369312 (51:5547)

[46] T. Tao. Norm convergence of multiple ergodic averages for commuting transformations. Erg. Th. Dynam. Sys., 28 (2008), 657-688. MR2408398(2009k:37012)

[47] T. Tao. The ergodic and combinatorial approaches to Szemerédi's theorem. Additive combinatorics, CRM Proc. Lecture Notes 43, Amer. Math. Soc., Providence, RI (2007), 145-193. MR2359471 (2008i:11017)

[48] T. Tao. The dichotomy between structure and randomness, arithmetic progressions, and the primes. Proceedings of the International Congress of Mathematicians, Madrid 2006, Vol. I, 581-608. MR2334204 (2009c:11025)

[49] T. Ziegler. Universal characteristic factors and Furstenberg averages. Jour. Amer. Math. Soc., 20 (2007), 53-97. MR2257397 (2007j:37004)

Department of Mathematics, Northwestern University, 2033 Sheridan Road, EvansTON, ILLINOIS 60208-2730

E-mail address: kra@math.northwestern.edu 